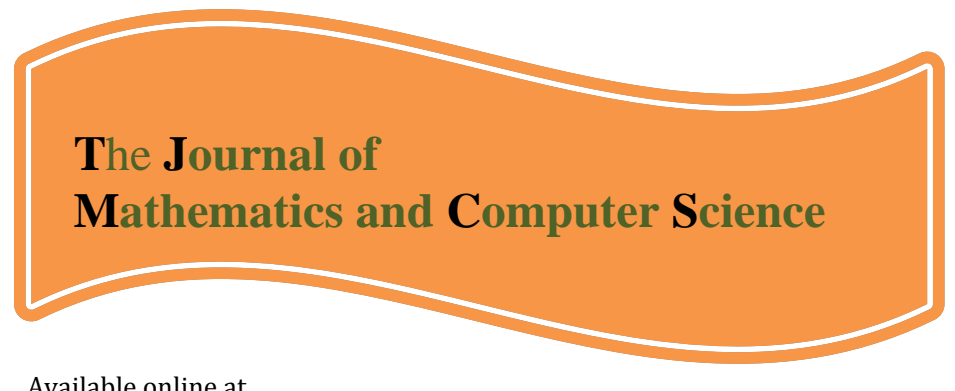

http://www.TIMCS.com

The Journal of Mathematics and Computer Science Vol .1 No.4 (2010) 313-320

\title{
A new approach to solve Multi-objective linear bilevel programming problems
}

\author{
M. H. Farahi1, ${ }^{*}$, E.Ansari² \\ Department of Applied Mathematics, Ferdowsi University of Mashhad, Farahi@math.um.ac.ir \\ Department of Mathematics, Islamic Azad University Mashhad-branch, Mashhad, Iran, ansari.nc@hotmail.com
}

Received: August 2010, Revised: October 2010

Online Publication: December 2010

\begin{abstract}
Many problems in sciences and industry such as signal optimization, traffic assignment, economic market,... have been modeled, or their mathematical models can be approximated, by linear bilevel programming (LBLP) problems, where in each level one must optimize some objective functions.

In this paper, we use fuzzy set theory and fuzzy programming to convert the multiobjective linear bilevel programming (MOLBLP) problem to a linear bilevel programming problem, then we extend the Kth-best method to solve the final LBLP problem. The existence of optimal solution, and the convergence of this approach, are important issues that are considered in this article. A numerical example is illustrated to show the efficiency of the new approach.
\end{abstract}

Keywords: Linear bilevel programming, Multi-objective linear bilevel programming, Fuzzy set theory, Fuzzy programming, Kth-best algorithm.

\section{Introduction}

\footnotetext{
${ }^{1,{ }^{*}}$ Professor in control and optimization

${ }^{2}$ MSc student in Dept of Math
} 
There are some methods for finding the global optimum of a bilevel programming problem in which both level's have just one objective function to be optimized. The majority of researchs on bilevel programming problems have been centered on the linear version of the problems such as Penalty function approach [1], Genetic algorithm [2,3], Grid-search algorithm [4] and the Kth-best algorithm [5].

The intent of this paper is to provide a new approach for solving multi-objective linear bilevel programming problems, so as mentioned, since we know some methods for solving BLP problems, we apply fuzzy set theory and fuzzy programming to convert our problem to a BLP problem, then we extened the classical Kth-best method, to find the global optimum solution of the achieved BLP problem. This algorithm computes global solution of linear BLP problems by enumerating extreme points of constraint region. We do not use the classical Kth-best algorithm [6] because of its deficiency, that is, it could not well solve LBLP problem when the upper level's constraints are in arbitrary linear form [7].

\section{Converting the multi-objective linear bilevel programming problem to a single- objective LBLP problem}

Consider a model in general as the following form:

$$
\begin{array}{ll}
\underset{x \in X}{\operatorname{Max}} & \left(c_{1}{ }^{t} x, c_{2}{ }^{t} x, \ldots, c_{k}{ }^{t} x\right) \\
\text { s.t } & A_{1} x+B_{1} y \leq b_{1} \\
\operatorname{Max}_{y \in Y} & \left(d_{1}{ }^{t} y, d_{2}{ }^{t} y, \ldots, d_{k^{\prime}}{ }^{t} y\right) \\
\text { s.t } & A_{2} x+B_{2} y \leq b_{2} \\
& x \geq 0, y \geq 0
\end{array}
$$

where

$$
c_{i} \in R^{n}, i=1, \ldots, k, d_{j} \in R^{m}, j=1,2, \ldots, k^{\prime}, x \in X \subseteq R^{n}, y \in Y \subseteq R^{m}, A_{1}, B_{1}, A_{2}, B_{2}
$$

are appropriate matrices. The upper level and the lower level are called leader and follower, respectively. $x$ is the leader's decision variable and $y$ is the follower's decision variable.

To change the MOLBLP problem (1) to a BLP problem, first find an upper and also lower bounds for any objective function in the first and second level of the MOLBLP problem (1). One may call $Z_{i}^{U}$ and $Z_{i}^{L}$, respectively as upper and lower bounds of $c_{i}^{t} x ; i=1,2, \ldots, k$ (objective functions of the first level) and $z_{j}^{U}$ and $z_{j}^{L}$ respectively as upper and the lower bounds of $d_{j}^{t} y, j=1,2, \ldots, k^{\prime}$ ( objective functions of the follower). To find these bounds, one may solve the following BLP problems (use the method explained in [8]).

$$
\begin{array}{cccc}
(\mathrm{LP})_{i} & (i=1, \ldots, k) & \operatorname{Max}_{x \in X} & c_{i}{ }^{t} x \\
& \text { s.t } & A_{1} x+B_{1} y \leq b_{1} \\
(\mathrm{LP})_{j} & \left(j=1, \ldots, k^{\prime}\right) & \operatorname{Max}_{y \in Y} & d_{j}{ }^{t} y \\
& \text { s.t } & A_{2} x+B_{2} y \leq b_{2} \\
& & x \geq 0, y \geq 0 .
\end{array}
$$

Let $\left(x_{1}{ }^{*}, y_{1}{ }^{*}\right), \ldots,\left(x_{k}{ }^{*}, y_{k}{ }^{*}\right)$ and $\left(x_{1}^{\prime *}, y_{1}^{\prime *}\right), \ldots,\left(x_{k^{\prime}}^{\prime *}, y_{k^{\prime}}^{\prime *}\right)$ be the solutions of the first and second level respectively. Define the following matrices: 


$$
A=\left[\begin{array}{cccc}
c_{1}{ }^{t} x_{1}{ }^{*} & c_{2}{ }^{t} x_{1}{ }^{*} & \ldots & c_{k}{ }^{t} x_{1}{ }^{*} \\
c_{1}{ }^{t} x_{2}{ }^{*} & c_{2}{ }^{t} x_{2}{ }^{*} & \vdots & c_{k}{ }^{t} x_{2}{ }^{*} \\
\vdots & \vdots & \ddots & \vdots \\
c_{1}{ }^{t} x_{k}{ }^{*} & c_{2}{ }^{t} x_{k}{ }^{*} & \ldots & c_{k}{ }^{t} x_{k}{ }^{*}
\end{array}\right] \quad \text { and } \quad B=\left[\begin{array}{cccc}
d_{1}{ }^{t} y_{1}^{\prime *} & d_{2}{ }^{t} y_{1}^{\prime *} & \ldots & d_{k^{\prime}}{ }^{t} y_{1}^{{ }^{*}} \\
d_{1}{ }^{t} y_{2}^{\prime}{ }^{*} & d_{2}{ }^{t} y_{2}^{\prime *} & \vdots & d_{k^{\prime}}{ }^{t} y_{2}{ }^{*} \\
\vdots & \vdots & \ddots & \vdots \\
d_{1}{ }^{t} y_{k^{\prime}}{ }^{*} & d_{2}{ }^{t} y_{k^{\prime}}^{\prime *} & \ldots & d_{k^{\prime}}{ }^{t} y_{k^{\prime}}{ }^{*}
\end{array}\right]
$$

The maximum and minimum values in each column of $A$ denoted respectively by $Z_{i}^{U}$ and $Z_{i}^{L}$, $(i=1, \ldots, k)$, also the maximum and minimum values in each column of $B$ denoted respectively by $z_{j}^{U}$ and $z_{j}^{L}, \quad\left(j=1, \ldots, k^{\prime}\right)$. The differences $Z_{i}{ }^{U}-Z_{i}{ }^{L} \quad$ and $\quad z_{j}{ }^{U}-z_{j}{ }^{L}$ are the constants of admissible violations. Now we can define a membership function corresponding to each level (leader and follower) for any fuzzy goal, as the following:

$$
\begin{aligned}
& \text { for } i=1, \ldots, k, \quad \mu_{i}\left(c_{i}{ }^{t} x\right)=\left\{\begin{array}{cc}
1 & c_{i}{ }^{t} x \geq Z_{i}{ }^{U} \\
\frac{c_{i}{ }^{t} x-Z_{i}{ }^{L}}{Z_{i}{ }^{U}-Z_{i}{ }^{L}} & Z_{i}{ }^{L} \leq c_{i}{ }^{t} x \leq Z_{i}{ }^{U} \\
0 & c_{i}{ }^{t} x \leq Z_{i}{ }^{L}
\end{array}\right. \\
& \text { for } j=1, \ldots, k^{\prime}, \quad \phi_{j}\left(d_{j}{ }^{t} y\right)=\left\{\begin{array}{cc}
1 & d_{j}{ }^{t} y \geq z_{j}{ }^{U} \\
\frac{d_{j}{ }^{t} y-z_{j}{ }^{L}}{z_{j}{ }^{U}-z_{j}{ }^{L}} & z_{j}{ }^{L} \leq d_{j}{ }^{t} y \leq z_{j}{ }^{U} \\
0 & d_{j}{ }^{t} y \leq z_{j}{ }^{L}
\end{array}\right.
\end{aligned}
$$

Since membership functions are the degrees of satisfaction, they must be maximized. So if we consider $\lambda_{1}=\min \left\{\mu_{1}, \ldots, \mu_{k}\right\}$ and $\lambda_{2}=\min \left\{\phi_{1}, \ldots, \phi_{k^{\prime}}\right\}$, then the MOLBLP problem (1) now changes to the LBLP problem (4) as follows:

$$
\begin{array}{ll}
\underset{x \in X, \lambda_{1}}{\operatorname{Max}} & \lambda_{1} \\
\text { s.t } & A_{1} x+B_{1} y \leq b_{1} \\
& \mu_{i}\left(c_{i}{ }^{t} x\right) \geq \lambda_{1}, \\
& 0 \leq \lambda_{1} \leq 1
\end{array}
$$




$$
\begin{array}{ll}
\underset{y \in Y, \lambda_{2}}{\operatorname{Max}} & \lambda_{2} \\
\text { s.t } & A_{2} x+B_{2} y \leq b_{2} \\
& \phi_{j}\left(d_{j}{ }^{t} y\right) \geq \lambda_{2}, \quad j=1, \ldots, k^{\prime} \\
& 0 \leq \lambda_{2} \leq 1 \\
& x \geq 0, y \geq 0
\end{array}
$$

By substituting the membership functions from (3), the LBLP problem (4) changes to:

$$
\begin{array}{ll}
\operatorname{Max}_{x \in X, \lambda_{1}} & \lambda_{1} \\
\text { s.t } & A_{1} x+B_{1} y \leq b_{1} \\
& c_{i}{ }^{t} x-\left(Z_{i}^{U}-Z_{i}{ }^{L}\right) \lambda_{1} \geq Z_{i}{ }^{L}, \\
& 0 \leq \lambda_{1} \leq 1 \\
& \lambda_{2} \\
\operatorname{Max}_{y \in Y, \lambda_{2}} & A_{2} x+B_{2} y \leq b_{2} \\
& d_{j}{ }^{t} y-\left(z_{j}{ }^{U}-z_{j}{ }^{L}\right) \lambda_{2} \geq z_{j}{ }^{L}, \\
& 0 \leq \lambda_{2} \leq 1 \\
& x \geq 0, y \geq 0
\end{array}
$$

Now we have a single-objective LBLP problem, in which, $x \in X \subseteq R^{n}$ and $\lambda_{1} \in[0,1]$ are the decision variables for the upper level and $y \in Y \subseteq R^{m}$ and $\lambda_{2} \in[0,1]$ are the decision variables for the lower level.

\section{Extended Kth-best algorithm}

Kth-best algorithm investigates in the constraint region of the LBLP problem to find the optimal solution at a vertex of this region. Now for applying the algorithm, we use the definitions expressed in $[7,9]$.

Constraint region of the LBLP problem (5) defines as follows: 


$$
\begin{aligned}
S= & \left\{\left(x, y, \lambda_{1}, \lambda_{2}\right) \mid A_{1} x+B_{1} y \leq b_{1}, c_{i}{ }^{t} x-\left(Z_{i}{ }^{U}-Z_{i}{ }^{L}\right) \lambda_{1} \geq Z_{i}{ }^{L}, A_{2} x+B_{2} y \leq b_{2},\right. \\
& \left.d_{j}{ }^{t} y-\left(z_{j}{ }^{U}-z_{j}{ }^{L}\right) \lambda_{2} \geq z_{j}{ }^{L}, 0 \leq \lambda_{1} \leq 1 \quad 0 \leq \lambda_{2} \leq 1, x \geq 0, y \geq 0, i=1, \ldots, k, j=1, \ldots, k^{\prime}\right\}
\end{aligned}
$$

Denote the projection of $S$ onto the leader's decision space by $S(X)$ and the feasible set for the follower for all $x, \lambda_{1} \in S(X)$, these two sets define as follows:

$$
S(X)=\left\{x \in X, \lambda_{1} \in[0,1]: \exists y \in Y, \exists \lambda_{2} \in[0,1],\left(x, y, \lambda_{1}, \lambda_{2}\right) \in S\right\}
$$

(constraints of the leader and the follower are satisfied.)

$$
S(x)=\left\{y \in Y, \lambda_{2} \in[0,1]:\left(x, y, \lambda_{1}, \lambda_{2}\right) \in S\right\}
$$

Then solving the following problem for any $x \in S(X)$, gets the follower's rational reaction set.

$$
\begin{aligned}
& \operatorname{Max}_{y \in Y, \lambda_{2}} \quad \lambda_{2} \\
& \text { s.t } \quad B_{1} y \leq b_{1}-A_{1} x \\
& c_{i}{ }^{t} x-\left(Z_{i}{ }^{U}-Z_{i}{ }^{L}\right) \lambda_{1} \geq Z_{i}{ }^{L}, \quad i=1, \ldots, k \\
& 0 \leq \lambda_{1} \leq 1 \\
& B_{2} y \leq b_{2}-A_{2} x \\
& d_{j}{ }^{t} y-\left(z_{j}{ }^{U}-z_{j}{ }^{L}\right) \lambda_{2} \geq z_{j}{ }^{L}, \quad j=1, \ldots, k^{\prime} \\
& 0 \leq \lambda_{2} \leq 1 \\
& y \geq 0
\end{aligned}
$$

We denote the set of the optimum solutions of the above problem by $P(x)$. Now we present the inducible region of the problem (5) by:

$$
I R=\left\{\left(x, y, \lambda_{1}, \lambda_{2}\right):\left(x, \lambda_{1}\right) \in S,\left(y, \lambda_{2}\right) \in P(x)\right\}
$$

To ensure that (5) has an optimal solution, one must consider the following assumptions:

(1) $S$ is nonempty and compact.

(2) $P(x)$ is nonempty, (i.e. $P(x) \neq \varnothing$ ). 
(3) $P(x)$ is a point to point map (otherwise some difficulties appear, which are explained in [10],[11]).

Thus in terms of the above definitions and notations, the LBLP problem (5) can be written as:

$\operatorname{Max}\left\{\lambda_{1}:\left(x, y, \lambda_{1}, \lambda_{2}\right) \in I R\right\}$

Every theorem for LBLP problems also satisfies for MOLBLP problems, since we made the LBLP problem (5) from MOLBLP problem (1), these two are equuivalent. The most important theorems are:

Theorem 1. If S is nonempty and compact, there exists an optimal solution for a linear BLP problem.

Theorem 2. A solution for the linear BLP problem occurs at a vertex of IR .

Theorem 3. The solution of the linear BLP problem occurs at a vertex of $S$.

Details of the proofs can be found in [7].

Now to solve (5), one can use the extended Kth-best algorithm as the following:

Step 1. Put $i \leftarrow 1$. Solve the problem $\operatorname{Max}\left\{\lambda_{1}:\left(x, y, \lambda_{1}, \lambda_{2}\right) \in S\right\}$ with the simplex method to obtain the optimal solution $\left(x_{[1]}, y_{[1]}, \lambda_{1[1]}, \lambda_{2[1]}\right)$. Let $W=\left\{\left(x_{[1]}, y_{[1]}, \lambda_{1[1]}, \lambda_{2[1]}\right)\right\}$ and $T=\varnothing$. Go to Step 2.

Step 2. Solve (6) by the assumption $x=x_{[i]}, \lambda_{1}=\lambda_{1[i]}$ with the simplex method to obtain the optimal solution $\left(\tilde{y}, \tilde{\lambda}_{2}\right)$. If $\left(\tilde{y}, \tilde{\lambda}_{2}\right)=\left(y_{[i]}, \lambda_{2[i]}\right)$, stop; $\left(x_{[i]}, y_{[i]}, \lambda_{1_{[i]}}, \lambda_{2[i]}\right)$ is the global optimum of (1). Otherwise go to Step 3.

Step 3. Let $W_{[i]}$ denote the set of adjacent extreme points of $\left(x_{[i]}, y_{[i]}, \lambda_{1[i]}, \lambda_{2[i]}\right)$ such that $\left(x, y, \lambda_{1}, \lambda_{2}\right) \in W_{[i]}$ implies $\lambda_{1} \leq \lambda_{1[i]}$. Let $T=T \bigcup\left\{\left(x_{[i]}, y_{[i]}, \lambda_{1[i]}, \lambda_{2[i]}\right)\right\} \quad$ and $\quad W=\left(W \bigcup W_{[i]}\right) \backslash T$. Go to Step 4.

Step 4. Set $i \leftarrow i+1$ and choose $\left(x_{[i]}, y_{[i]}, \lambda_{1[i]}, \lambda_{2[i]}\right)$ so that $\lambda_{1[i]}=\operatorname{Max}\left\{\lambda_{1}:\left(x, y, \lambda_{1}, \lambda_{2}\right) \in W\right\}$. Go to Step 2.

\section{Numerical example}

Consider the following problem with $x \in R^{2}, y \in R^{2}$ and $X=\{x \geq 0\}, Y=\{y \geq 0\}$.

$$
\begin{array}{ll}
\underset{x \in X}{\operatorname{Max}} & \left(x_{1}+2 x_{2}, 3 x_{1}+x_{2}\right) \\
\text { s.t } & x_{1}+x_{2} \leq 3
\end{array}
$$




$$
\begin{array}{cl}
\underset{y \in Y}{\operatorname{Max}} & \left(y_{1}+3 y_{2}, 2 y_{1}+y_{2}\right) \\
\text { s.t } & -x_{1}+y_{1}+y_{2} \leq 6 \\
& -x_{2}+y_{1} \leq 3 \\
& x_{1}+x_{2}+y_{2} \leq 8
\end{array}
$$

The problem is equivalent to the following problem by fuzzy programming.

$$
\begin{array}{ll}
\underset{x \in X, \lambda_{1}}{\operatorname{Max}} & \lambda_{1} \\
\text { s.t } & x_{1}+2 x_{2}-3 \lambda_{1} \geq 3 \\
& 3 x_{1}+x_{2}-6 \lambda_{1} \geq 3 \\
& x_{1}+x_{2} \leq 3 \\
& 0 \leq \lambda_{1} \leq 1 \\
& \\
& \\
\operatorname{Max}_{y \in Y, \lambda_{2}} & \lambda_{2} \\
& \\
\text { s.t } & y_{1}+3 y_{2}-12.5 \lambda_{2} \geq 8.5 \\
& 2 y_{1}+y_{2}-10 \lambda_{2} \geq 7 \\
& -x_{1}+y_{1}+y_{2} \leq 6 \\
& -x_{2}+y_{1} \leq 3 \\
& x_{1}+x_{2}+y_{2} \leq 8 \\
& 0 \leq \lambda_{2} \leq 1
\end{array}
$$

By appling the extended Kth-best algorithm, the optimal solution of the problem reached at the point $\left(x_{1}^{*}, x_{2}^{*}, y_{1}^{*}, y_{2}^{*}, \lambda_{1}^{*}, \lambda_{2}^{*}\right)=(1.5,1.5,4.1154,3.3846,0.5,0.4615)$.

Then objective function values are: for the upper level functions $x_{1}+2 x_{2}=4.5,3 x_{1}+x_{2}=6$ and for the lower level functions $y_{1}+3 y_{2}=14.2692,2 y_{1}+y_{2}=11.6154$.

\section{Conclusion}

We found the global optimal solution of the multi-objective linear bilevel programming problems under fuzzy programming and extended Kth-best algorithm. If the constraint regions of the upper level and lower level be non-empty and compact we are able to solve any linear multi-objective bilevel programming problems.

It might suggest that this approach can be extended for non-linear problems where the leader's objective function is quadratic. 


\section{References}

[1] G. Anandalingam and D. J White, "A penalty function approach for solving bilevel linear programs" , Journal of Global Optimization,,Vol. 3, No. 4, pp. 397-419, 1993.

[2] B. Liu, "Stackelberg-Nash equilibrium for multi-level Programming with multi-follows using genetic algorithms", Computers math applications,Vol. 37, No. 7, pp. 79-89, 1998.

[3] R. Mathieu, L. Pittard and G. Anandalingam, "Genetic algorithm based approach to bilevel linear ptogramming", R. A. I. R. O Researche operationelle,Vol. 28, pp. 1-21, 1994.

[4] J.F. Bard, "A grid search algorithm for the linear bilevel programming problem", 14-th Annual Meeting of American Institude for Decision Science, San Francisco, CA.2, pp. 256-258, 1982.

[5] W. F. Bialas and M. H. Karwan, "On two level optimization"., IEEE Transactions on Automatic Control, Vol. 27, No. 1, pp. 211-214, 1982.

[6]W. Bialas , M. Karwan, J. Shaw, "A parametric complementary pivot approach for two-level linear programming", Technical Report, 80-2, state university of New York at Buffalo, Operations Research programs, 1980.

[7]J. Lu, C. Shi, G. Zhang, "An extended Kth-best approach for linear bilevel programming", Applied Mathematics and computation, Vol. 164, pp. 843-855, 2005.

[8]H. J. Zimmerman, " Fuzzy programming and linear programming with several objective functions", Fuzzy sets and systems, Vol. 1, 45-55, 1978.

[9]J. Lu, C. Shi, G. Zhang, "On the definition of linear bilevel programming solution", Applied Mathematics and Computation, Vol. 160, pp. 169-176, 2005.

[10] J. F. Bard, "Practical Bilevel Optimization Algorithms and Applications",Kluwer academic Publishers, Dordrecht, Boston, London, 1998.

[11]S. Dempe, "Foundations of Bilevel Programming", Kluwer Academic Publishers, Dordecht, Boston, London, 2002 . 